\title{
The case for informal spaces in the workplace
}

\author{
Monica Biagioli \\ University of the Arts London, England
}

\begin{abstract}
This chapter makes the case for informal spaces in the workplace by emphasising the qualitative benefits they can provide, most specifically in the realm of innovation. This goes against the current bias in evidence-based policy that the most valued evidence is that which can be measured (Belfiore \& Bennett, 2008, p.5) and aligns with qualitative forms of representing experience, knowledge, and outcomes. The link is made here between transformation and innovation. Innovation is seen as the fertile offshoot of creating a space for growth, debate and experimentation; one parallel to and overlapping with the more formal channels of established communication and interaction in the workplace. Transformation is difficult to substantiate and requires qualitative forms of expression and analysis to come into view. This chapter aims for a more balanced view of all forms of analysis and argues that qualitative forms are the most effective at capturing innovation.
\end{abstract}

Keywords: Relational, Qualitative Analysis, Transformative, Quantitative Bias, Living Organism, Movement, Creative Collision, Tacit Communication, Complete Experiences

\section{INTRODUCTION}

The focus of this chapter is to reframe the question of management and organisational practices in the workplace from the point of view of the people engaging with the systems and processes. There are a huge number of discussions and debates around the quantifiable aspects of delivering innovation through the framework of the workplace: how spaces are organised and how communication flow is managed within the work context. Elements such as efficiency and cost are factored into decisions regarding allocation of space, time and access to various staff. This focus on measurable components belies a bias for quantification.

This chapter aims to make the case for informal spaces in the workplace by emphasising the qualitative benefits they can provide, most specifically in the realm of innovation. This goes against the current bias in evidence-based policy that the most valued evidence is that which can be measured (Belfiore \& Bennett, 2008, p.5) and aligns with qualitative forms of representing experience, knowledge, and outcomes. The link is made here between transformation and innovation. Innovation is seen as the fertile offshoot of creating a space for growth, debate and experimentation; one parallel to and overlapping with the more formal channels of established communication and interaction in the workplace. Transformation is a key word here and one that Belfiore and Bennett flag as highly contentious because it is difficult to substantiate (Belfiore \& Bennett, 2008, p.5). Allowing for that difficulty, this chapter will aim to make the case that quantitative forms of analysis have an inherent bias, and that this bias is equally if not more 
problematic than the inability for qualitative forms of analysis to provide evidence that can be measured. This chapter aims for a more balanced view of all forms of analysis and argues that qualitative forms are the most effective at capturing innovation.

\section{BACKGROUND}

The current bias for quantification is the result, as Eleonora Belfiore and Oliver Bennett outline in their intellectual history of cultural policy, of a growing reliance on evidence to make the case for policy. This took form in the 1990's as a pragmatic response to what was then viewed as ideologically driven decision-making. Decisions would be based on effectiveness rather than political bias. Elements would be measured against one another in order to ascertain the best way forward. In an effort to make the case for evidence-based decision-making, "hard data, such as facts, trends and survey information, were widely seen as the 'gold standard' " (Belfiore \& Bennett, 2008, p.5). Quantitative forms of analysis took over as they provided a way of measuring and comparing which sated the need to base decisions on clearlyarguable numerical frameworks. If experiences and spaces can be reduced to numerical units, comparisons can be more readily made, and more importantly, argued. This approach was all about making the case and the decision was made to base it on scientific methods as those had the most credibility. The subtext riding below the surface of this approach was (and, it is argued, continues to be) that qualitative forms of analysis cannot be trusted to formulate decisions.

\section{MAIN FOCUS OF THE CHAPTER}

\section{Issues, controversies, problems}

\section{WORK AS EMBODIED EXPERIENCE}

In his book The value of arts for business, Giovanni Schiuma set out an approach for Arts-based Initiatives within the workplace that addressed this imbalance from the perspective of the organisation as a living organism: "Organisations are continuously challenged to find new routes to accomplish their strategic business objectives and to deliver value to stakeholders. They have to become agile, intuitive, imaginative, flexible to change and innovative. This means that organisations have to be managed as 'living organisms' in which the people and the organisational aesthetic dimensions are recognised as fundamental factors to meet the complexity and turbulence of the new business age." (Schiuma, 2011, p.2) It is precisely that agility and flexibility that is a concern in the current structures of work across sectors and disciplines. Agility and flexibility imply movement, and movement is increasingly being restricted throughout and within management frameworks. Yet this is an essential factor in the creation of management structures that can truly respond as living organisms.

When considering agility and flexibility, it is best to look at the in-between spaces and the pockets of interaction across and through organisational frameworks, rather than within them, in order to allow for 
collision of ideas, hybrid solutions, and synthesis, all of which are the hallmarks of innovative thinking. For this Edward T. Hall's diagram of human activity is quite pertinent. Hall devised this framework to express how cultural understandings and changes move from informal, to formal, to technical knowledge absorption, but this is quite relevant in discussions on innovation. He deduced a system of understanding human activity in three porous layers: formal, informal, and technical (Hall, 1959). The formal layer is occupied by expectations, values and structures; those values and structures are shaped into more codified forms (rituals, language, protocols) in the technical layer; and the informal layer is where shifts can happen and is the terrain of gesture, play, informal learning, and a sense of individual space and beliefs. The informal is where collisions can happen. There, what is codified into practice from a formal belief system, comes into contact with individual beliefs and approaches and can then be converted, through technical analysis, into formal structures. Hall states, "Since man progresses from formal belief to informal adaptation and finally to technical analysis, a theory of change is also implied in this tripartite division which is at the heart of my theory." (Hall, 1959, p.51) The mode of conveying these shifts is referred to as a silent language by Hall to bring to light the fact that these ways of being and interactions are most often not explicit but tacit.

Applying Hall's model to account for the key dimensions of work organisation and structure: work spaces and hierarchies represent the formal layer, established communication protocols and procedures operate on the technical layer, and times and places outside of the formal and technical structures (having a tea, stepping out together with a colleague for some air) embody and activate the informal layer. As more and more tools and practices are put in place to measure the efficiency of workers and spaces, there is less attention and less incentive to engender informal spaces and interactions. Or, more specifically, those settings are created within the formal structures already established, so not encouraging flow from different layers of the organisation but reinforcing the layers already in place. This reflexive looping of knowledge transmission, not only ossifies knowledge but it also has the effect of making communications and transmissions outside of the formal layer disembodied.

In his discussion on embodiment, R.D. Laing does not place a value judgement on embodied versus unembodied. "What we have are two basic existential settings. The difference in setting does not preclude every basic issue, good and bad, life and death, identity, reality and irreality, from arising in the one context as in the other, but the radically different contexts in which they occur determine the basic ways in which they are lived." (Laing, 1960, p.69) Laing was talking about schizophrenia at the time, but this discussion becomes even more relevant now on a societal level as we have to make decisions about how important embodiment is to our notion of being human. "The embodied person... has as his starting-point an experience of his body as a base from which he can be a person with other human beings." (Laing, 1960, p.69) In his definition of experience, John Dewey took embodiment as a given: "Experience is the result, the sign, and the reward of that interaction of organism and environment which, when it is carried to the full, is a transformation of interaction into participation and communication." (Dewey, 1934, p.22) For Dewey, there were complete experiences, holding transformative and aesthetic potential and characterised by feelings of satiety and fulfilment, and inchoate experiences, obstructed by distraction and dispersion (Dewey, 1934, p.35). The link between Laing and Dewey is a discussion around human existence that is an embodied experience. 
As the world of work evolves to make room for robotic components and algorithmic computation to input into decision-making and realise more and more complex tasks, it is increasingly important to consider the organisation's view of their workers as embodied persons. To what an extent is the work flow considered on a holistic level to take into consideration the fullness or incompleteness of the experience for the workers involved? If the transformative potential (innovation) is dependent on full experiences, should this not be a much more prominent consideration? Belfiore and Bennett's caution about transformative potential plays a strong role here. As the work system aims to integrate more and more computationally derived inputs, it necessitates the reduction of human experience to components that can be measured against and combined with them so as to make the system operations more efficient. This has the effect of undermining the possibility of full experiences. Transformation is difficult to quantify (make the case) and as a result the agility and flexibility that makes an entity a living organism (Schiuma, 2011) is increasingly sacrificed to make it possible for the complex system parts to work together operationally.

As Michael Polanyi states, " if we build up a culture recklessly on the assumption that only things are valid which can be broken into parts - and that the putting together will take care of itself - we may be quite mistaken, and all kinds of things may follow." (Polanyi, 1989, quoted in Gill, 2015). When there are no methods or structures in place to allow for actual human contact across layers, other communications are necessarily broken down into quantifiable formats (data sets, statistics, form filling) so that all the possible embodied tacit information transmission is lost, broken down as it is into its constituent parts. It is a situation where "the paradigm of data (of parts) and utility gives primacy to transactional information over that which is relational." (Gill, 2015) Anne Pässilä and Allan Owens state it further: "This obsession with measurement is locking us deeper and deeper in our intra-organisation silos. The measurement system does not include for example how often we co-operate with colleagues from other units, when we cross boundaries and take risks.” (Pässilä and Owens, 2016)

In the book Tacit Engagement, psychologist Satinder Gill addresses this key problem by proposing a relational interface. In her study on evolving human-computer interfaces, she raises the issue of quantification in relation to current technological advances so vast that they render the human into constituent parts in order to interact with computational elements. She addresses "the problems of bottlenecks of vast quantities of data and how to relate to them (expert systems, data bases, big data), and how to support our relations with each other and share and enable us to impart knowledge and skills when we are distributed in space via various mediating interfaces". Michael Polanyi's notion of a "personal act of knowing", referencing tacit knowledge - tradition, inherited practices, implied values, and prejudgments - as a crucial part of scientific knowledge, again reinforces the position set forth by Hall. Gill explains it further: " the explicit always has a tacit dimension (Rosenbrock 1988, Wittgenstein 1958), and the tacit can be shared in silence with someone who understands us very well, where you become one, a state of I Thou (Buber 1923), or where you share the same background of knowledge where the utterance of a seeming 'explicit representation' says it all.” (Gill, 2015)

The informal, not easily accountable through data, is then the domain of embodied tacit information transmission. This type of activity happens at the edges of the points of connection. For example, a chance meeting in the hallway lets two colleagues who don't know each other by face actually meet in 
person and discuss a problem that has required multiple email communications and incorrect input of data. Their five minute chat, with accompanying non-verbal cues, allows them to explore a range of options and set a solution in motion that had reached standstill via disembodied forms of contact. It is the informal nature of the setting as well that allows for the communication to flow more readily, unrestricted as it is by the requirements of procedure and protocol in the office. But it is the tacit, non-verbal cues that will be the most fruitful in imparting knowledge and understanding. It is what Gill refers to as "knowledge as skilled embodied performance" (Gill, 2015). It requires trust in the performative act of interaction with another as a different level of communication, beyond the verbal, to impart the sense of truth and judgement that the person possesses about that situation.

A combination of vernacular forms of discussion and interaction set that type of innovation in motion. But this is a difficult position to support in a context where formal structures have been set that work on the assumption that, as Polanyi states, "only things are valid which can be broken into parts". If an experience is not quantifiable, does that render it invalid? If a quick glance and a word with a colleague sets your mind at rest about the progress of an idea, or even more excitingly, changes the course of your work to find a new method to solve the problem, can that be written up in a report? Be part of the analysis and findings? And even more challenging, if in order to move from informal to technical (in order to achieve formal recognition), the tacit understanding needs to be broken down into constituent parts because that is the only way to prove validity, are we not denying the tacit element of the experience?

The question here is, what is the role of quantification in this case? To ignore what is not visible in the data and its analysis? What if the incorrect data is being collected? What if the analysis follows a procedural thread established through formal structures and ignores what is not visible because the right question has not been asked yet? (Pässilä and Owens, 2016)

Tacit forms of expression and engagement happen perhaps at the edges of the points of connection. The in-between areas of engagement across departments and across disciplines are much more difficult and complicated to capture as data bits that can be accounted for and tracked, as those contributions are tacit rather than explicit and more complicated to trace to direct sources as it is a combination of vernacular forms of discussion and interaction that set those innovations in motion. They are better served by qualitative forms of recording and analysis. Their embodied shape can be retained through subjective forms of expression that retain a sense of human experience and interaction: traces of the essence of an experience, for example, and narrative structure to give the subjective form and meaning.

\section{A SERIES OF INCHOATE EXPERIENCES}

What is driving this fear of what cannot be objectively accounted for? It is the terrain of philosophy, sociology and psychology to deal with the mental structures of experience and consciousness, as those areas of inquiry deal with the embodied and the sensed in human experience, both in groups and as individuals. But as technology embeds more visibly into our relational experiences, the desire to understand such matters leads more often than not to breaking things down into forms that can be mediated through the technological interfaces we use to communicate with one another. Technological 
interfaces work with elements that can be transmitted through the protocols established for the benefit of data transmission, so communication is reduced in order to make transmission possible, or shaped to fit the required form.

Jeremy Holmes addresses this impasse in his review of The Master and His Emissary: The Divided Brain and the Making of the Western World by Iain McGilchrist. He lists the various maladies of our modern and post-modern dilemma as diagnosed by McGilchrist: "loss of tolerance of ambiguity; the carrying out of procedures by rote without understanding; de-individualisation; paranoia and lack of trust; a worship of the quantitative and devaluation of quality; and downgrading of expertise and its capacity to react with spontaneity and creativity in favour of 'expert' knowledge that can be pre-determined."(Holmes, 2012, p.163)

In the book, McGilchrist presents his thesis on the differences between right and left brain hemisphere, stressing that there has been an increasing dominance by left hemisphere activity at the expense of right brain function. "Our brains", he states, "not only dictate the shape of the experience we have of the world, but are likely themselves to reflect, in their structure and functioning, the nature of the universe in which they have come about." (McGilchrist, 2007, p.460) This thesis has been hotly debated since the book was published and it is not for this chapter to enter that specific debate but to draw from some of the key reflections and conclusions aptly made by McGilchrist about the human condition now and how it is shaped by reinforced biases.

For him it is a question of attention. The left hemisphere, interested in manipulation and representation, sets its focus on directed tasks clearly identified through completion and achievement. The right hemisphere, focused on presence and understanding, uses all the information available to it, including environmental factors and previous experience, to make overall judgements about a situation or a problem. The left hemisphere seeks certainty while the right hemisphere allows for ambiguity. It is his position that the right hemisphere perceives and guides action as a reverberation, while the left hemisphere follows through with the action and represents the right hemisphere's judgement as enacted form in space and time. Here, it is not so important whether it is left or right hemisphere but more what those key functions are: presence, understanding, judgement, ambiguity, reverberation; and the role they are allowed to play in workplace tasks and decision-making.

There are some key skills connected to right hemisphere activity: capacity for attention, contextualisation, being present, empathy, allowing for a state of 'betweenness'. These are then 'handed' over to the left hemisphere for classification, verbal analysis, comparison with previous experience and 'difference coding' (Northoff). "Once processed in this way, experience is then returned to the Right hemisphere so that a living, creative engagement with the world can continue." (Holmes, 2012, p.165) But in McGilchrist's view this is not how things are shaping. Instead, increasingly, the human capacity to absorb and consider experience, even if it is not formed yet (connected by McGilchrist to right hemisphere function), is being undermined by the capacity to prove as fact (connected by McGilchrist to left hemisphere function and by this author to quantification). 
Many of the maladies diagnosed by McGilchrist resonate when considering the trajectory of work and where the emphasis is being placed for many, if not most, in the workplace: automatisation of the workplace with workers plugging into procedures by rote; little tolerance for the role of doubt in decisionmaking; quantitative forms of analysis valued highly in industry, business and education at the expense of qualitative methods; and reliance on pre-determined knowledge while downgrading judgement based on spontaneity and creativity. These developments do not favour the people working in the systems. In many cases there is a serious degradation of quality of work experience as a result of many if not most work practices being implemented across a wide range of work environments. As McGilchrist argues, this has been developing consistently since the Industrial Revolution.

Back in 1995, author Kurt Vonnegut was interviewed for his views about technological progress, and this is what he had to say:

"We're always trying to replace jobs. Keeping lists, taking inventory, those are all things to do with life. And then somebody comes along and says, "Hey, you don't need to do that anymore." Well, thanks, but how the hell am I supposed to support my family? You, you silly fool, you've still got a job, sure. There's this great word that the British use all the time: redundant. Workers are declared redundant. How'd you like to come into this world and be told you're redundant? Built into human beings is a need, which nobody bothers to even acknowledge, to do something useful. But instead of worrying about what human beings need, we worry about what machines need. There's no talk at all about what human beings are deprived of; all the talk is about what industries are being deprived of." (Schafer, 1995)

The recent surge of technological breakthroughs, combining increasingly complex learning algorithms with sophisticated robotics and emergent biomimicry, amplifies the meaning of Vonnegut's statement even further. Huge numbers of industries are investing in research that will wipe out jobs. As Wired magazine reported in October 2015: "The South Korean government has handed Samsung a multi-million dollar investment to develop factory robots that can carry out complex tasks normally reserved for nimble human fingers. The country's Ministry of Trade, Industry and Energy said the $\$ 16.75$ billion won $(\$ 14.8 \mathrm{~m}, £ 9.57 \mathrm{~m})$ investment would help manufacturers compete with cheap labour in China.”

(Temperton, 2015)

South Korean workers' unions held a mass rally in April 2015 "demanding... that the Government halts plans to overhaul the country's labour market, which they say will benefit only the employers in dealing with labour issues" (Suk, 2015). The government's response six months later came as shocking news. It invested over $\$ 16$ billion to replace workers with robots and handed over the funds to a private company, Samsung. For a democratically-elected government that holds public funds and its public's trust to invest in the divestiture of its citizens' ability to provide for themselves and their families is an admission that decisions made at the government level are concerned with macro considerations at the expense of micro outcomes. In other words, South Korea's battle to take business away from China is more important than maintaining a job structure for its citizenry.

The recent developments in South Korea are one case in point, but this is more the rule than the exception across the developed world. It has worrying implications as it sets a precedent that winning at the 
quantification stakes on the macro level is by far more important than maintaining societies that are good for the citizens of that country. A government that is willing to invest $\$ 16$ billion to wipe out massive numbers of jobs held by its own citizenry, without due consideration for how that investment could create new and better jobs that are equal in number if not greater than before, is admitting that its citizenry is not its main priority. As Vonnegut would say, "There's no talk at all about what human beings are deprived of; all the talk is about what industries are being deprived of."

How can we not be aware of this trajectory and fight to do something about it? Media representations have had a very strong role to play in shaping our way of communicating and our sense of self (McLuhan, 1964). The many layers of diversion and entertainment broadcast from various media sources create a level of inertia that Daniel Boorstin identified over 50 years ago as a visual gauze, at the time when mass media lodged into the North American consciousness. He visualised it as a mirage impeding people from engaging with the reality of their social condition. (Biagioli, 2015a, p.12) His solution? "Each of us must disenchant himself, must moderate his expectations, must prepare himself to receive messages coming from the outside... The least and most we can hope for is that each of us may penetrate the unknown jungle of images in which we live our daily lives. That we may discover anew where dreams end and where illusions begin. This is enough. Then we may know where we are, and each of us may decide for himself where he wants to go." (Boorstin, 1961, p.260) This disjunct between what is communicated (and how) and what is experienced leads then to more frequent inchoate experiences; dispersed and incomplete.

From an organisational and management perspective, the process can be aided by making the relationship between macro (systems) and micro (people working in the systems) visible and, therefore, relatable. Or, the lack of interaction can be made visible. McGilchrist argues potently that increasingly our world is being shaped and organised to account for experience through quantifiable forms at the expense of ambiguity, spontaneity, creativity, doubt and the holistic judgement that emerges as a result of those functions. This lack of balance in assessing that which can be proven as fact and that which is to be considered through a variety of factors is having serious repercussions in our society, as human creativity functions are downgraded at the expense of 'hard' data. One way to begin to address this is by making that misalignment of value visible to people.

\section{SOLUTIONS AND RECOMMENDATIONS}

\section{CREATING RELATIONAL SPACES}

The informal level of interaction, where creative collisions can happen, is where the relationship between systems and the people working in the systems can be made visible. Through tacit forms of communication including gesture, informal spaces engender a sense of play and encourage informal types of learning which allow for an individual sense of space and beliefs to come into contact or collision with the systems in place in the workplace. 
On the informal level, terms such as holistic, participatory, organic, osmosis, and intuitive become operative. Holistic expresses a concern with complete systems rather than with individual parts: looking at interactions and interdependencies across elements within the systems. Intuitive refers to what is learned or understood from the environment and culture; an area where cultural activities can have a real impact. Osmosis is a process where information is diffused and absorbed via assimilation in space. Through osmosis, the intuitive reinforces innate feelings that people have, without relying on rational thinking and analysis. The organic is a process that occurs in living organisms, responsive to change and accounting for the interconnected whole, so the responses to change are done holistically. In terms of participatory, this is a key word when considering how the sense of informality arises in a particular space. Sustainable becomes the overarching term here to focus plans for the future and consider our requirements as human beings within the systems being developed. (Biagioli, 2015b, p.12-13)

The first problem with tacit forms of communication in informal spaces is that the definitions of these words in themselves set up the problem of accountability. How do you measure something that is holistic, intuitive, and impacted by osmosis? It is quite difficult. These terms lend themselves to qualitative analysis and are difficult to capture by quantitative means. There are urban researchers looking at the potential harm it causes to attempt to quantify activities that are essentially non-measurable. Davisi Boontharm, part of the Measuring the non-Measurable research project at Keio University, Japan spoke at the Mapping Culture Conference in Coimbra, Portugal in May 2014 about mapping urban intensities and the importance of the subjective in that activity. The argument there is that the subjective cannot be specified as data points to be plotted on a graph. The subjective is beyond measured data. (Boorntham, 2014)

Thus, it becomes important to advocate for qualitative means of analysis in allocating informal spaces and assessing their value for the function of innovation in the workplace. Otherwise their capacity as innovation drivers becomes under-represented in relation to quantifiable elements such as cost and efficiency. Qualitative methods can account for value in the longterm, assessing the viability of innovation as sustainable solutions to the system in place; functions such as tentative exploration, the ability to doubt current frameworks in order to devise new ones, allowing for a state of 'betweenness', and the application of judgement based on experience and tacit understanding of context can all come into play in problem solving. This would address one of the key maladies of contemporary society identified by McGilchrist: relying on pre-determined knowledge while downgrading judgement based on spontaneity and creativity.

Ad hoc activity in informal spaces, generally perceived as non-measurable and resistant to quantification, can be viewed in this way as having a qualitative effect that can be expressed through various representations and outcomes. The case then needs to be made for qualitative forms of analysis in order to protect the function of ad hoc activity in informal spaces in the innovation process.

In informal spaces what is codified into system beliefs and structures in the formal level can come into direct contact with individual notions that can help shift the system to reflect better its participants in the organisation framework and communication structure. This can be best reflected and communicated through qualitative research and analysis that involves art and design approaches. 
In her work on relational interfaces, Gill emphasises the importance of design as an intervention: "a 'local forcing move', a 'juxtaposition that stresses rhythm', or 'two or more events mixing to reveal hybrid natures.' There is no hierarchy, only interdependence in this 'template' of the Informal." She stresses the core function of art and design activities to allow for thinking that is "In Between, Betwixt and In Between, Betweeness, Hybrid, Transitional, Liminal, Transcendent". (Gill, 2015) This type of thinking is the realm of right hemisphere function and can help sustain perceptual and mental processing functions under-represented by the left hemisphere bias currently in place in the construction and application of work flow and spaces. In the process of art and design activities, there is a strong correlation between making and doing, which reinforces a healthy interaction of functions that McGilchrist relates to left and right brain hemisphere. If the activities are tactile, then they also reinforce our sense of being in physical space, and connect our thinking processes to the functions of our bodies in space, enhancing the wellbeing of the individuals involved as well as supporting system innovation.

Value is a key concern of this chapter. As society places more and more importance on explicit factors and rewards those who can break problems down to components that can be quantified and therefore monetised, there are few incentives for attempting to provide a holistic view of the problem that can guide the use of those components in ways that are better for society as a whole, and that can, therefore, provide social value.

This trend is reinforced by the seamless integration of our digital technology to our lives lived in the physical world, where we break down our activities as components that can be transmitted and communicated through the interfaces we are creating. "As Mark Zuckerberg repeatedly emphasised during the 2014 F8 conference keynote, Facebook apps play an essential role in Facebook Inc.'s newest expansion strategy - that is, to become a 'cross-platform platform' (Zuckerberg et al., 2014) that connects not just friends, family and acquaintances but the millions of platforms, websites, 'stacks' and services that currently constitute the web." (Kant, 2015)

If we perceive ourselves to be simply one more platform, along with other web services, then Laing's notion that "the embodied person... has as his starting-point an experience of his body as a base from which he can be a person with other human beings" (Laing, 1960, p.69) can no longer be taken for granted to define our sense of self. Here Marshall McLuhan's notion that the medium is the message is very pertinent as the means we are creating to communicate our sense of being is itself reshaping and reconfiguring our notion of who we are, our sense of identity. In a similar way, McGilchrist envisions the evolution of the brain hemispheres to reflect the current world, mostly shaped by left hemisphere, and warns of the dangers of undermining right hemisphere activity which is greatly concerned with presence and embodied context.

The primary means of our current communication technology is sight, and the importance placed on it is increasing as our dependence on communication mediated through technology is increasing. This allows for seamless integration of our human functions as just another window, just another platform, as Sherry Turkle so presciently foresaw in her 1996 book Life on the screen: identity in the age of the internet. Art and design activities that can reconnect multi-sensory experiences become even more crucial for maintaining our sense as embodied selves. These types of activities, integrating thinking with making, 
allow for the tentative exploration and play so critical in transformative processes that lead to new ways of perceiving the world and that shape advances of new methods in the workplace.

\section{THE ABILITY TO DOUBT}

If we allow that innovation does not arise out of certainty but through tentative and speculative efforts to reconfigure elements previously understood in one particular form so as to synthesise material and knowledge into new shapes, or in new directions, or with new goals, then this is the domain of right hemisphere activity. Current forms of organising people and spaces leave increasingly less room for playful exploration of the material without a clearly identified goal. What is required is the space and time for notions to emerge from a process and to lead to new ways of looking at a problem or for transforming the way we approach a situation.

Presence - a state of awareness situating the individual in time and space - is achieved through physical sensation and experience allowing physical sensation to function as psychic anchor (Moore, 2007, p.97). It activates the ability of the spatial framework to provide a sense of history from which to embrace the shifts and changes of time.

In a 1967 review of Understanding Media: The extensions of man, Richard Kostelanetz wrote: "[Marshall McLuhan] maintains that a major shift in society's predominant technology of communications is the crucially determining force behind social changes. Initiating great transformations not only in social organization but human sensibilities." (Kostelanetz, 1967) The current digital system allows us to slot in and, as a result, fragmentation of thinking is taking place with greater frequency as people experience disjunct between their digital identities and their experiences in physical reality. (Biagioli, 2015a)

Now that integration of digital with physical is increasingly becoming more seamless, it is time to begin to incorporate other senses more consciously as a way of transmitting information. This would begin to correlate the digital to the physical more meaningfully, allowing for more integral responses and minimising fragmentation of activities and thought processes. In The Point of Being, authors de Kerckhove and Miranda call for "a re-sensorialization of the environment" so as "to complement the visually biased perspective with a renewed sense of our relationship to the spatial and material surrounds" and encourage a "topological reunion of sensation and cognition". They identify "a visual dominance among the senses people use to perceive the world as a whole, a trend that has repressed or toned down information from other senses." (Kerckhove and Miranda, 2014, p.2)

Information from other senses can be understood as tacit embodied knowledge and the research team of Allan Owens and Anne Pässilä have developed an understanding of knowledge production that has it as its basis. They identify art and art pedadogy approaches as the best means to connect tacit knowledge with reflection that can lead to innovation in the workplace. Based on previous discussion in this chapter, these activities can be enacted best in informal spaces and can then be organised as findings to be implemented within the system. 
Art and design approaches work best because these activities can "become disencumbered of the governing configuration of power and open an alternative modality of relations" (Ziarek, 2004, p.3). For this purpose as well informal spaces work best. As Krzysztof Ziarek describes it, "art becomes a dynamic occurrence, a shifting and movable field of tensors and forces capable of repeatedly and differently rupturing the social conditions of production and reception"(2004, p.20). Ziarek applauds art's ability to “open an alternative modality of relations"(2004, p.3) and, so, the perfect conditions for innovative practice are created: dynamic, shifting, movable, rupturing and open.

It is in this milieu that tentative exploration can take place and allow for what Gill describes as a fundamental function: the ability to doubt. "Technologies of data and utility presume to represent and provide certainty and in the case of expertise this can cause experienced professionals to lose confidence in their ability to doubt (Goranzon 1988, 1992) and thereby in their confidence to make judgements, as the ability to doubt is fundamental to our capacity to judge with confidence. As a result, workers are not encouraged to judge but to accept what output the computer provides them with and may potentially lose their critical capacities over time. The certainty of data abstracted from our experience has implications for a range of interactive technologies that represent human behaviours, including gesture and emotion, and ideas about how we think." (Gill, 2015)

Thus, the work circumstances described earlier in the chapter can in this way be disrupted to good end. "The use of arts in organisations operates from within the tension of this paradox, providing ways for organizational actors to define the right questions rather than rushing in to give solutions" (Pässilä and Owens) It is the combination of informal settings and art's ability to access an "alternate modality of relations" (Ziarek, 2004) that make art and design approaches critical for articulating "questions that make problems visible that we are perhaps not yet aware of" (Pässilä and Owens, 2016). This ability is an essential aspect of innovative thinking.

\section{FUTURE RESEARCH DIRECTIONS}

\section{A STATE OF BETWEENNESS}

A key objective of this chapter is to set out a means to reinforce our sense of place to reconnect us to the lives we live in the physical world and to reinforce our embodied experience and all our physical senses, not just sight. This would allow for a rebalancing between the transactional and the relational (quantitative and qualitative) and would encourage information transmitted beyond sight to play a much stronger role in decision-making. Tacit embodied knowledge, in particular intuition and the ability to doubt, would allow qualitative judgement to play a central role in emergent ideas and future planning; all in the terrain of innovation.

This in itself would be a huge gain. Currently, the ability to make assessments is increasingly being removed from the organisational framework, replaced by precisely calculated guidelines of how to proceed through a particular set of circumstances or problems. And, so, the employee's ability to make independent judgements is increasingly being constrained, if not removed entirely from the equation. The 
essential functions of experience and knowledge expressed as judgement are replaced by a looping circularity of procedural thinking reinforcing itself in the formal level. This in turn is removing the potential for innovation to emerge out of problem-solving activities.

There are huge benefits and potential from the evolution of technology up to this point, and in many ways it has improved people's lives for the better. But we are at a juncture now where the scales might be tipping in the opposite direction and dependence on technology to solve and communicate is potentially diminishing our capacity to question, challenge and take a holistic view of a situation. What is being lost as a result is the trust placed on the individual to make judgements and to apply skill and knowledge to solve an old problem in a new way. Furthermore, Gill believes that "flow between real life and the virtual is dissolving in 'hyper existence', and the key to full and reciprocal interconnectedness is that it is a full bodied and creative experience." (Gill, 2015) This echoes Schiuma's understanding of the organisation as a living organism, requiring agility, intuition, imagination and flexibility in order to respond to the complexity of today's business environment and to the tremendous human challenges that we face.

Creative interventions in the form of art and design activities in informal spaces at work can help redress the balance. They can help make people's connections to their local environments more palpable, foregrounding the sensed alongside the visual; break down the formal structures; create an alternate form of relations; and allow for a re-alignment of the physical with the mental processes. In this way some critical capacities currently on the wane could be reinstated, including capacity for attention, contextualisation, being present, empathy, and allowing for a state of 'betweenness'.

\section{CONCLUSION}

This chapter has argued for the application of art and design techniques in informal spaces in the workplace to address the problem of the current imbalance between the transactional and the relational in problem-solving. It has been argued that the transactional has taken over decision-making functions as a result of the formal structures in place that accommodate technological advances at the expense of human relational needs. Once in place, this imbalance reinforces itself in increasingly tighter loops through procedures and protocols and undermines the ability of individuals and groups to innovate within the organisational framework. The question here is, are organisations willing to devote space and time for activities that do not have a clearly set out objective within the goals of the organisation? Are they willing to create a physical space of in-between engagement across different layers of the organisation to allow for connections to take place that are not possible within the organisational structure?

What is proposed is the creation of an alternate space, a third space in close proximity to or within a work environment, where art and design activities can take shape through a forming process of workshops leading to exhibitions. This means that the thinking and making processes remain in the same space. Typically in an exhibition context, preparations are made in sites other than the exhibiting space. Here, it is critical that all activities related to exhibited works take place in the same context so as to reinforce one another. The site becomes a playground, but one potent with possibilities of addressing concerns and problems, whether large or small, tackled by those working in the environment. It can also be envisioned 
as a site of contestation, where alternate practices to those in place can emerge and be tested, where new products and objects can emerge for consideration, where voices seldom heard can have their platform. This would necessarily require a 'letting go' by management in terms of expected approaches or outcomes, and could be seen more as a creative collision of multiple ideas; an art and design incubator of notions moving from the transactional to the relational to be enacted in space. This approach would require bravery, but any innovation that has transformative potential requires that.

\section{REFERENCES}

Belfiore, E. \& Bennett, O. (2008). The social impact of the arts: an intellectual history. Basingstoke: Palgrave Macmillan.

Biagioli, M. (2015a). Zones of intensity invested with desire. Kyлтура/Culture, (12), 7-15. Retrieved March 15, 2016, from http://journals.cultcenter.net/index.php/culture/article/view/177/161

Biagioli, M. (2015b). Fine art installations: expressions of cultural identity and catalysts for intuitive business models. In The 7th Art of Management \& Organization Conference Papers (ISBN: 978090193116 0, pp. 12-21). York: University of York.

Biagioli, M. (2015c). Zones of intensity: reinforcing a sense of place. In 10th International Forum on Knowledge Asset Dynamics (IFKAD). Culture, Innovation and Entrepreneurship: Connecting the knowledge dots. Bari: IFKAD.

Boontharm, D. (2014, May). Drawing as mapping in the search of urban quality. Conference talk presented at Mapping Culture: Communities, Sites and Stories Conference, Coimbra, Portugal.

Boorstin, D. J. (1961). The image: a guide to pseudo-events in America. New York: Atheneum.

Dewey, J. (1934). Art as experience. London: George Allen \& Unwin Ltd.

Gill, S. P. (2015). Tacit engagement: beyond interaction. London: Springer.

Hall, Edward T. (1959). The silent language. New York: Doubleday, New York. p.51[Ref 1]

Holmes, J. (2012). The master and his emissary. Psychoanalytic Psychotherapy, 26(2), 160-170. Retrieved November 20, 2015. 
Kant, T. (2015). FCJ-180 'spotify has added an event to your past': (re)writing the self through facebook's autoposting apps. FCJ The Fibreculture Journal, (25), 30-62.

Kerckhove, D.D. \& Miranda, D.A. (2014). The point of being. Newcastle upon Tyne: Cambridge Scholars Pub.

Kostelanetz, R. (1967, January 29). Understanding McLuhan (in part). Retrieved March 15, 2016, from http://www.nytimes.com/books/97/11/02/home/mcluhan-magazine.html

Kupfer, J.H. (1983). Experience as art: aesthetics in everyday life. Albany: State University of New York Press.

Laing, R. D. (1960). The divided self. New York: Pantheon Books.

McLuhan, M. (1964). Understanding media: the extensions of man. London: Ark.

Moore, N. (2007). Valorizing urban heritage? Redevelopment in a changing city. In Moore, N. and Whelan, Y. (eds.) Heritage, memory and the politics of identity: new perspectives on the cultural landscape. Aldershot: Ashgate.

Pässilä, A. \& Owens, A. (2016). Sensible sensitivity: arts pedagogy in management development. In Peter Stokes, Neil Moore, Simon M Smith, Caroline Rowland, Peter Scott (eds.) Organizational Management: Approaches and Solutions. Kogan Page.

Schafer, S. (1995, December 15). Vonnegut and Clancy on technology. Retrieved November 25, 2015, from http://www.inc.com/magazine/19951215/2653.html

Schiuma, G. (2011). The value of arts for business. Cambridge: Cambridge University Press.

Suk, L. Y. (2015, April 24). South Korean union workers rally against labour reforms. Retrieved November 25, 2015, from http://www.channelnewsasia.com/news/asiapacific/south-koreanunion/1805256.html

Temperton, J. (2015, October 19). Samsung is developing robots to replace cheap Chinese labour (Wired UK). Retrieved November 25, 2015, from http:/www.wired.co.uk/news/archive/2015-10/19/samsung-south-korea-robots-cheap-labour

Turkle, S. (1996). Life on the screen: identity in the age of the internet. London: Weidenfeld \& Nicolson.

Ziarek, K. (2004). The force of art. Stanford, CA: Stanford University Press. 


\section{KEY TERMS}

Transformation: the use of this term in the organisational context is in the realm of innovation, allowing for one type of activity, procedure, product or service to be completely reconsidered and reshaped into a new form, perhaps even with a new function all together.

Quantitative bias: quantification, requiring numerical data or data sets, is a reductive function that breaks the whole into segments that can be compared. The results are taken as fact more often than not because they are constructed out of data. What is not questioned is how much data, who collected it, how it was collected, and did it actually reflect the experience of the people and places being assessed. This is where potential bias can come into focus.

Embodied experience: the expectation that an experience is realised through the entire body, and so the experience is embodied. It is not just a mental thought process or a procedural communication that does not require full engagement in order to complete the procedure.

Tacit communication: expression that is sensed more than heard through a multi-sensory experience including spatial cues, body language and movement, and sonic responses such as tone of voice. This is in the qualitative realm, very difficult to quantify this type of communication.

Inchoate experience: the reference is to incomplete experiences and within the organisational and management context that could point to a number of expected interactions throughout the day, including inputting data into a system where the person inputting is not able to use human judgement but must follow a very strict set of protocols that limit the range and type of communication allowed.

State of betweenness: another term for this could be liminality, where it is allowed that the person in that state or location is between locations or states. It is argued in this chapter that this state is critical at key junctures in the innovation process to allow for new connections and hybrids to emerge into shape.

The right question: Pässilä and Owens argue potently that in innovation and problem solving the key is to create the right conditions for the right questions to emerge. Often in the workplace it is expected to come into an innovation situation with a question already in mind. The key to this approach is to assume that innovation is always a potential as long as the correct conditions are in place, and then the right questions can emerge. So the focus then becomes to create the conditions that make that possible. 